\title{
ON KANNAN MAPS
}

\section{CHI SONG WONG ${ }^{1}$}

ABSTRACT. Let $K$ be a (nonempty) weakly compact convex subset of a Banach space $B$. Let $T$ be a self map on $K$ such that for all $x, y$ in $K,\|T(x)-T(y)\| \leq(\|x-T(x)\|+\|y-T(y)\|) / 2$. It is proved without the continuity of $T$ and Zorn's lemma that $T$ has a fixed point if and only if $\inf \{\|x-T(x)\|: x \in K\}=0$. A characterization of the existence of fixed points for such $T$ is obtained in terms of close-to-normal structure. As consequences, the following results are obtained: (i) $T$ has a unique fixed point if $B$ is locally uniformly convex or more generally if $B$ has the property A: For any sequence $\left\{x_{n}\right\}$ in $B,\left\{x_{n}\right\}$ converges to a point $x$ in $B$ if it converges weakly to $x$ and $\{\|x\|\}$ converges to $\|x\|$; (ii) $T$ has a unique fixed point if $B$ is separable.

1. Introduction. Let $K$ be a weakly compact convex subset of a Banach space $B$. Let $T$ be a Kannan map on $K$, i.e. $T$ is a self map on $K$ such that for all $x, y$ in $K$,

$$
\|T(x)-T(y)\| \leq(\|x-T(x)\|+\|y-T(y)\|) / 2 .
$$

If $K$ has normal structure [3], we have found explicitly (in terms of \|\| ) all such $T$ [14]. For example, even for the simple case where $K$ is the unit interval $[0,1]$ in the real line $R$ with the usual norm, there are $2^{c}$ such $T$, among which, $c$ of them are nonexpansive $(\|T(x)-T(y)\| \leq\|x-y\|$, $x, y \in K)$ and $c$ of them are monotone, where $c$ is the cardinal of $R$. For the fixed point theory of such $T$, R. Kannan proved [6] that $T$ has a fixed point if $B$ is reflexive and if for any convex $T$-invariant subset $H$ of

Received by the editors July 3, 1973.

AMS(MOS) subject classifications (1970). Primary 47H10; Secondary 54H25.

Key words and phrases. Close-to-normal structure, diametral point, fixed point, locally uniformly convex Banach space, separable Banach space, weakly compact convex set.

${ }^{1}$ This research was partially supported by the National Research Council of Canada, grant A8518; it was prepared while the author was at the Summer Research Institute at Carleton University and at the Séminaire de mathématiques supérieures (fixed point theory and its applications) at the Université de Montréal. 
$K(T(H) \subseteq H)$ with more than one point, $\sup \{\|y-T(y)\|: y \in H\}<\delta(H)$, where $\delta(H)$ is the diameter of $H$. P. Soardi proved [12] that $T$ has a fixed point if either $T$ has diminishing orbital diameters [2] or $K$ has normal structure [3]. Other earlier results can be found in the references of [6] and [12]. Since $K$ has normal structure-if $B$ is uniformly convex [5] or $K$ is compact [3], $T$ has a fixed point if $B$ is uniformly convex or $K$ is compact. In [14], the author combined all of the above results into the following more general result: $T$ has a (unique) fixed point if for any closed convex $T$-invariant subset $H$ of $K$ with more than one point, $\inf \{\|x-T(x)\|$ : $x \in H\}<\delta(H)$.

Since there may be uncountably many such $H$, the above result is not practical enough. In this paper, it is proved without the continuity of $T$ and without the use of Zorn's lemma that $T$ has a unique fixed point if (and only if) either

(i) $\inf \{\|x-T(x)\|: x \in K\}=0$, or

(ii) for any $x$ in $K$ with $\|x-T(x)\|>0,\|x-T(x)\|>\|y-T(y)\|$ for some $y$ in $K$.

By using this result and our result in [14] mentioned above, we prove that every Kannan map on $K$ has a unique fixed point if $K$ has a close-to-normal structure: for any closed convex subset $H$ of $K$ with $\delta(H)>0$, there exists $x$ in $H$ such that

$$
\|x-y\|<\delta(H), \quad y \in H
$$

( $x$ may be a diametral point of $H: \sup \{\|x-y\|: y \in H\}=\delta(H)$ ). It is obvious that $K$ has a close-to-normal structure if it has normal structure. In [1], a weakly compact convex subset $H$ of a reflexive separable Banach space which has no normal structure is given. However, this $H$ contains a point $x$ for which $\|x-y\|<\delta(H), y \in H$. In [15], we shall prove that $K$ has a close-to-normal structure if either (i) $B$ is separable or (ii) $B$ is lo-. cally uniformly convex [10], or more generally, $B$ has the property $A$ : For any sequence $\left\{x_{n}\right\}$ in $B,\left\{x_{n}\right\}$ converges to a point $x$ in $B$ if $\left\{x_{n}\right\}$ converges weakly to $x$ and $\left\{\left\|x_{n}\right\|\right\}$ converges to $\|x\|$. Since weakly compact convex subsets of a separable Banach space may not have normal structure [1], our result is actually more general than the above result of Soardi. Comparing our results with those of Kannan in [6], we emphasize here that our results are so general that we nowhere assume that the given Banach space is reflexive, or the given weakly compact convex set $K$ has normal structure, or the given Kannan map on $K$ is continuous. 


\section{Main results.}

Theorem 1. Let $K$ be a weakly compact convex subset of a normed linear space. Let $T$ be a Kannan map on $K$. Then the set $\{\|x-T(x)\|$ : $x \in K\}$ has a smallest number.

Proof. Let $r>0$. Consider the set $K_{r}$ defined by $K_{r}=\{y \in K: \| y-$ $T(y) \| \leq r\}$. Let $I=\left\{r \in[0, \infty): K_{r} \neq \varnothing\right\}$. Since $K$ is bounded, $I \neq \varnothing$. It suffices to prove that $I$ has a smallest element or equivalently, $\bigcap_{r \in I} K_{r} \neq \varnothing$. For each $r$ in $I$, let $H_{r}$ be the closed convex hull of $T\left(K_{r}\right)$. Since for any $r, s$ in $I$ with $r<s, K_{r} \subseteq K_{s}$, the family $\mathcal{F}=\left\{H_{r}: r \in I\right\}$ has the finite intersection property. Since each $H_{r}$ is closed and convex, it is weakly closed. So by the weak compactness of $K, \mathcal{F}$ has nonempty intersection. It then suffices to prove that $H_{r} \subseteq K_{r}$ for each $r$ in $I$. Let $r \in I, y \in H_{r}, \epsilon>0$. Then there exist $t_{1}, t_{2}, \ldots, t_{n}$ in $[0,1]$ and $y_{1}, y_{2}, \ldots, y_{n}$ in $K_{r}$ such that $\sum_{i=1}^{n} t_{i}=1$ and $\left\|y-\sum_{i=1}^{n} t_{i} T\left(y_{i}\right)\right\|<\epsilon$. Thus

$$
\begin{aligned}
\|y-T(y)\| & \leq\left\|y-\sum_{i=1}^{n} t_{i} T\left(y_{i}\right)\right\|+\left\|\sum_{i=1}^{n} t_{i} T\left(y_{i}\right)-T(y)\right\| \\
& <\epsilon+\sum_{i=1}^{n} t_{i}\left\|T\left(y_{i}\right)-T(y)\right\|<\epsilon+\sum_{i=1}^{n} t_{i} \frac{\left\|y_{i}-T\left(y_{i}\right)\right\|+\|y-T(y)\|}{2} \\
& \leq \epsilon+(r+\|y-T(y)\|) / 2 .
\end{aligned}
$$

So

$$
\|y-T(y)\| \leq 2 \epsilon+r \text {. }
$$

Since $\epsilon>0$ is arbitrarily chosen, $\|y-T(y)\| \leq r$, i.e. $y \in K_{r}$. Hence $H_{r} \subseteq$ $K_{r}$.

Theorem 2 below follows easily from the above theorem.

Theorem 2. Let $K$ be a weakly compact convex subset of a normed linear space. Let $T$ be a Kannan map on $K$. Then the following conditions are equivalent:

(a) $T$ has a unique fixed point.

(b) $\inf \{\|y-T(y)\|: y \in K\}=0$.

(c) For any $x$ in $K$ with $\|x-T(x)\|>0$,

$$
\|x-T(x)\|>\|y-T(y)\| \text { for some } y \text { in } K .
$$


Theorem 3. Let $X$ be a weakly compact convex subset of a normed linear space $B$. Let $T$ be a Kannan map on $K$.

(a) Suppose that $T$ has no fixed point. Then there exists a $T$-invariant closed convex subset $K$ of $X$ such that $\delta(H)>0$ and $\|T(x)-x\|=\delta(H)$ for all $x$ in $H$.

(b) Suppose that for any closed convex $T$-invariant subset $H$ of $X$ with $\delta(H)>0$, there exists $x$ in $H$ for which $\|x-y\|<\delta(H), y \in H$. Then $T$ has a unique fixed point. In particular, $T$ has a unique fixed point if $K$ has a close-to-normal structure.

Proof. (a) By Zorn's lemma, there exists a minimal nonempty $T$-invariant closed convex subset $K$ of $X$. By Theorem 1 , there exists $x$ in $K$ such that

$$
\|x-T(x)\|=\inf \{\|y-T(y)\|: y \in K\} .
$$

Let $r_{0}=\|x-T(x)\|$. By hypothesis, $r_{0}>0$. We shall now use the same notation as in our proof for Theorem 1 . Since $H_{r_{0}} \subseteq K_{r_{0}}$,

$$
T\left(H_{r_{0}}\right) \subseteq T\left(K_{r_{0}}\right) \subseteq \operatorname{cl~} c_{0} T\left(K_{r_{0}}\right)=H_{r_{0}}
$$

By minimality of $K, H_{r_{0}}=K$ and therefore $K_{r_{0}}=K$. By the choice of $r_{0}$, $\|y-T(y)\|=r_{0}$ for all $y$ in $K$. Now for any $y, z$ in $K_{r_{0}}$,

$$
\|T(y)-T(z)\| \leq(\|y-T(y)\|+\|z-T(z)\|) / 2=r_{0} .
$$

So $\delta\left(T\left(K_{r_{0}}\right)\right) \leq r_{0}$. Therefore, $\delta(K)=\delta\left(H_{r_{0}}\right)=\delta\left(T\left(K_{r_{0}}\right)\right) \leq r_{0}$. Thus $\delta(K)=$ $r_{0}$. Hence

$$
\|y-T(y)\|=\delta(K) \text { for all } y \text { in } K \text {. }
$$

(b) follows from (a).

Theorem 4. Let $B$ be a normed linear space. Then the following two propositions are equivalent:

(a) Every weakly compact convex subset of B has a close-to-normal structure.

(b) Every Kannan map on a weakly compact convex (nonempty) subset of $B$ has a unique fixed point.

Proof. (a) $\Rightarrow$ (b). Apply Theorem 3.

(b) $\Rightarrow(a)$. Suppose to the contrary that there exists a weakly compact convex subset $K$ of $B$ which has no close-to-normal structure. Then $K$ contains a weakly compact convex subset $H$ such that $\delta(H)>0$ and for 
any $x$ in $H$, there exists an element $T(x)$ in $H$ for which $\|x-T(x)\|=$ $\delta(H) . T$ is a Kannan map on $H$ which has no fixed point, a contradiction to our hypothesis.

3. Remarks and examples. Let $K$ be a weakly compact convex subset of a Banach space $B$. There are two related open problems concerning the existence of fixed points for self maps on $K:$ (i) Every nonexpansive self map on $K$ has a fixed point. (ii) Every Kannan map on $K$ has a fixed point. Theorem 4 drops the above open problem (ii) out of the fixed point theory and adds an open problem to the geometry of Banach spaces: Every weakly compact convex subset of a Banach space has a close-to-normal structure. Because of this progress, we would like to compare the fixed point theory for nonexpansive maps and the fixed point theory for Kannan maps. Examples will be given to support our point of view.

$1^{\circ}$. Both nonexpansive maps and Kannan maps can be described in simple geometrical terms. For example, $T$ is a Kannan map on $K$ if and only if there exist $a_{1}, a_{2}$ in $[0,1]$ such that $a_{1}+a_{2}=1$ and

$$
\|T(x)-T(y)\| \leq a_{1}\|x-T(x)\|+a_{2}\|y-T(y)\|
$$

for all $x, y$ in $K$, i.e. if we think of $T$ as a motion, then the distance $\|T(x)-T(y)\|$ between $x$ and $y$ after the motion is no greater than the average $a_{1}\|x-T(x)\|+a_{2}\|y-T(y)\|$ of the distances $\|x-T(x)\|,\|y-T(y)\|$ moved by $x$ and $y$. It is then not too surprising that the following two similar results are obtained: (a) $T$ has a fixed point if $T$ is a nonexpansive self map on $K$ and if $K$ has normal structure [9]. (b) $T$ has a fixed point if $T$ is a Kannan map on $K$ and if $K$ has normal structure [12]. In fact, these results can be proved for a family of maps: If $K$ has normal structure, then every commuting family of nonexpansive (Kannan) self maps on $K$ has a common fixed point [9]. However, it is not known that every nonexpansive self map on $K$ has a fixed point if $K$ has a close-to-normal structure.

$2^{\circ}$. Every nonexpansive map is uniformly continuous. In fact every family of nonexpansive self maps on $K$ is equicontinuous. This fact is important to the existence of a common fixed point for a family of maps [11]. Kannan maps, however, may be continuous only at their fixed points. For example, the map $T$ defined by

$$
T(x)= \begin{cases}1-x & \text { if } x \in[0,1] \text { and if } x \text { is irrational, } \\ (1+x) / 3 & \text { if } x \in[0,1] \text { and if } x \text { is rational, }\end{cases}
$$


is a Kannan map on the unit interval and is continuous (nonexpansive) only at its fixed point $x_{0}=1 / 2$. In fact, when $K$ is the unit interval, there are $2^{c}$ Kannan maps which are not continuous. So it is of importance to see if every discontinuous Kannan map on $K$ has a fixed point. For example, does every lower (upper) semicontinuous Kannan map on $K$ have a fixed point?

$3^{\circ}$. Every Kannan map has at most one fixed point. However a nonexpansive map may have more than one fixed point. Also, the family of nonexpansive self maps on $K$ is a monoid with composition as its multiplication. The following example shows that the family of all Kannan maps on $K$ may not even be a semigroup: Let $T$ be the map on the unit interval defined by $T(x)=1-x, x \in[0,1]$. Then $T$ is a Kannan map. However, $T^{2}$ is the identity map on $[0,1]$ which is obviously not a Kannan map. The above observations suggest that it makes much more sense to investigate the common fixed points for a family, in particular a semigroup, of nonexpansive maps than to investigate the common fixed points for a family of Kannan maps [8].

$4^{\circ}$. If $T$ is a nonexpansive self map on $K$, then by considering the maps $T_{z, t}$ 's with

$$
T_{z, t}(x)=(1-t) z+t T(x), \quad x, z \in K, \quad t \in(0,1),
$$

one can conclude from the Banach contraction mapping theorem that

$$
\inf \{\|x-T(x)\|: x \in K\}=0 \quad[13] \text {. }
$$

Thus the difficulty of obtaining a fixed point for nonexpansive self maps $T$ on $K$ is the existence of $\min \{\|x-T(x)\|: x \in K\}$. From Theorem 1 we know there is no such difficulty for Kannan maps. The difficulty of obtaining a fixed point for a Kannan map on $K$ is to show that $\inf \{\|x-T(x)\|: x \in K\}=$ 0 . It may be worthwhile to point out here that $T_{z, t}$ 's may not be Kannan maps even if $T$ is: Let $T$ be the map on the unit interval defined by

$$
T(x)= \begin{cases}1-x & \text { if } x \in[0,1 / 3) \\ (x+1) / 3 & \text { if } x \in[1 / 3,1]\end{cases}
$$

Then $T$, but not $T_{0,1 / 2}$, is a Kannan map.

\section{REFERENCES}

1. L. P. Belluce, W. A. Kirk and-E. F. Steiner, Normal structure in Banach spaces, Pacific J. Math. 26 (1968), 433-440. MR 38 \#1501.

2. L. P. Belluce and W. A. Kirk, Fixed-point theorems for certain classes of nonexpansive mappings, Proc. Amer. Math. Soc. 20 (1969), 141-146. MR 38 \#1663. 
3. M. S. Brodskiǐ and D. P. Mil'man, On the center of a convex set, Dokl. Akad Nauk SSSR 59 (1948), 837-840. (Russian) MR 9, 448.

4. D. F. Cudia, Rotundity, Proc. Sympos. Pure Math., vol. 7, Amer. Math. Soc., Providence, R. I., 1963, pp. 73-97. MR 27 \#106.

5. M. M. Day, Normed linear spaces, 2nd rev. ed., Ergebnisse der Mathematik und ihrer Granzgebiete, N. F., Heft 21, Springer-Verlag, Berlin, 1962. MR 26 \#2847.

6. R. Kannan, Fixed point theorems in reflexive Banach spaces, Proc. Amer. Math. Soc. 38 (1973), 111-118.

7. W. A. Kirk, A fixed point theorem for mappings which do not increase distances, Amer. Math. Monthly 72 (1965), 1004-1006. MR 32 \#6436.

8. Anthony T. Lau and Chi Song Wong, Common fixed points for a semigroup of mappings, Proc. Amer. Math. Soc. 41 (1973), 223-228.

9. Teck-Cheong Lim, A fixed point theorem for families of nonexpansive mappings (submitted).

10. A. R. Lovaglia, Locally uniformly convex Banach spaces, Trans. Amer. Math. Soc. 78 (1955), 225-238. MR 16, 596.

11. Theodore Mitchell, Common fixed-points for equicontinuous semigroups of mappings, Proc. Amer. Math. Soc. 33 (1972), 146-150.

12. P. Soardi, Su un problema di punto unito di S. Reich, Boll. Un. Mat. Ital. (4) 4 (1971), 841-845. MR 46 \#741.

13. Chi Song Wong, Fixed point theorems for nonexpansive mappings, J. Math. Anal. Appl. 37 (1972), 142-150. MR 45 \#2691.

14.

Fixed points and characterizations of certain maps, Pacific J. Math. (to appear).

15. - Close-to-normal structure and its applications, J. Functional Analysis (to appear).

DEP ARTMENT OF MATHEMATICS, UNIVERSITY OF WINDSOR, WINDSOR, ONT ARIO CAN ADA 